\title{
Parametric and Optimization Studies on the entrapment of Potassium Fertilizer into Chitosan-Poly(methacrylic acid) Carrier via Ionic Gelation
}

\author{
Diana Jane M. Plofino ${ }^{1}$, Clint Charles P. Brutas ${ }^{1}$, Catalino G. Alfafara ${ }^{1}$, Monet Concepcion Maguyon-Detras ${ }^{1}$, and \\ Veronica P. Migo ${ }^{1 *}$ \\ ${ }^{1}$ Department of Chemical Engineering, College of Engineering and Agro-Industrial Technology, University of the Philippines Los \\ Baños, Los Baños, Laguna 4031, Philippines
}

\begin{abstract}
Nanofertilizer is an emerging technology for exhibiting slow release mechanism of fertilizer application. This slow release mechanism allows increase in nutrient uptake of plants while minimizing environmental pollution; specifically, reducing eutrophication in bodies of water. This study includes parametric and optimization studies for ionic gelation process in the formulation of potassium fertilizer in chitosan polymethacrylic acid (CS-PMAA) carrier, and subsequent characterization of the formulated $\mathrm{K}$ fertilizer. A $2 \mathrm{k}$ factorial experimnental design was initially implemented to determine significant factors. Results show that polymerization time inversely affects the K content concentration of the K-CS-PMAA fertilizer due to the swelling behavior of chitosan, while K:CS-PMAA ratio directly affects the $\mathrm{K}$ content concentration. Upon numerical optimization, the conditions found to maximize $\mathrm{K}$ content of the formulated fertilizer are $3000 \mathrm{ppm} \mathrm{K}^{+}$corresponding to 1.5:1 ratio of the $\mathrm{K}$ loading concentration to CS-PMAA carrier for 30 mins polymerization time. The optimum $\mathrm{K}$ content of K-CS-PMAA fertilizer is about $34.98 \% \mathrm{w} / \mathrm{w}-$ less than the $44.27 \% \mathrm{w} / \mathrm{w} \mathrm{K}$ content of the fertilizer grade, muriate of potash (MOP). The Dynamic Light Scattering (DLS) and Scanning Electron Microscope (SEM) results of $368.1 \mathrm{~nm}$ and $75.4 \mathrm{~nm}$, respectively, indicated that KCS-PMAA is nanosized. The Fourier Transform Infrared Spectroscopy (FT-IR) results proved the presence of CS-PMAA with deviations at 1483.01 and 1405.07 caused by the vibration in the --COO-anion groups of PMAA indicating the attachment of potassium in the nanoparticle. Furthermore, the fertilizer formulated was proved to exhibit slow release behavior with the value of $83.70 \% \mathrm{~K}^{+}$release after 48 hours compared to the $99.43 \%$ release of MOP.
\end{abstract}

\section{Introduction}

Environmental problems associated with the excessive use of fertilizer to increase crop production to ensure food security prompted researchers to find alternative ways to increase efficiency of fertilizer application. Commercial fertilizers are being introduced to the soil for plant uptake thereby promoting enhanced plant growth. However, about $40-70 \%$ nitrogen, $80-90 \%$ phosphorus, and $50-90 \%$ potassium content are being lost to the environment and only the remaining percent reaches the plant [4]. This inefficient excessive application of fertilizers resulted to degradation of soil quality and eutrophication of lakes and rivers. The nutrients released from fertilizers compete with other organisms such as plants and fish on the dissolved oxygen in the water resulting to fish kill and other effects prohibiting plant growth. And for the air, the excess nitrogen in the soil contributes as greenhouse gas once released in the atmosphere in the form of nitrous oxide.

Nanotechnology, specifically the production of nanofertilizer, offers solution to this problem by utilizing smart fertilizer delivery system which can be slow release or controlled release. This involves reduction of the fertilizer size into nano-scale allowing the nutrients to enter even in the plant pores and resulting to more efficient nutrient use and less fertilizer loss to the environment (Siresena et al., 2013). Furthermore, with the growing interest of "less land area farming" such as vertical farming and hydrophonics, nanofertilizer studies provide a leap for the development of such method by allowing increased retention of the nutrients needed by the plants in the medium of farming e.g. water.

Nanofertilizer studies commonly involves entrapment of NPK fertilizers in inorganic carriers such as zeolites. Studies on the incorporation of NPK in organic carriers are still limited. Preliminary studies on the formulation of NPK fertilizers with CS-PMAA carriers were made by Abdel-Aziz et al. and Corradini et al. However, their study showed that $\mathrm{N}$ and $\mathrm{P}$ do not entrapped well in CSPMAA. Thus, to maximize the incorporation of the carrier, $\mathrm{K}$ fertilizer in the form of MOP will be entrapped alone in this study. 
Potassium is one of the macronutrients needed for plant growth and it is primarily responsible for increasing the crop's resistance to drought and disease, strengthening its stalk and straw, and improving the quality of its grain and seed. Generally, the source of potassium is potash which can be in the form of potassium chloride, potassium sulfate, potassium- magnesium sulfate, potassium thiosulfate and potassium nitrate. Deficiency of potassium is identifiable by the presence of brown or yellow spot in the plants leaves.

This nutrient will be entrapped in the CS-PMAA nanoparticle, a commonly used drug carrier and made of chitosan and methacrylic acid. Chitosan is a derivative of chitin which comes the exoskeleton or waste crab or shrimp shells. It is composed of polycation (CS) and polyanion (PMAA), with its free multiple - $\mathrm{COO}^{-}$groups it allows attachment of the $\mathrm{K}^{+}$in its surface and the nano gaps between the attachment of CS to PMAA incorporates $\mathrm{K}^{+}$inside the particle.

This study aimed to develop and optimize the process conditions for the entrapment of potassium fertilizer into a nanocarrier, CS-PMAA. Specifically, the objectives of the study are to determine the effects of the formulation parameters: KCl-CS-PMAA ratio $(\mathrm{K}$ loading concentration), polymerization time, and polymerization temperature; maximize the significant parameters, and; characterize the formulated K-CS-PMAA fertilizer.

\section{Materials and methods}

\subsection{Materials and reagents}

Muriate of Potash (MOP) served as the potassium source for the formulation of the potassium-CS-PMAA fertilizer. Chitosan with $85 \%$ deacetylation was used to formulate the nanoparticle and it was purchased from Xi'an Lyphar Biotech Co. Ltd. Reagents used were analytical grade, methacrylic acid (MAA) containing 250ppm MEHQ and potassium persulfate $\left(\mathrm{K}_{2} \mathrm{~S}_{2} \mathrm{O}_{8}\right)$ were purchased from Sigma-Aldrich.

\subsection{Experimental design}

The number of runs and combination of the varied parameters were determined using Design Expert $^{\circledR}$ Software Version 11. Using $2 \mathrm{k}$ factorial design, polymerization time, polymerization temperature, and $\mathrm{KCl}$ :CS-PMAA ratio were varied and evaluated based on their significant effects on total $\mathrm{K}$ content of the formulated K-CS-PMAA fertilizer formulated, which served as the response. Statistical analysis was performed using ANOVA at $95 \%$ confidence interval to determine the significance of factors considered.

The significant factors were then optimized following Central Composite Design of Response Surface Methodology. Numerical optimization was done to determine the conditions that maximizes total $\mathrm{K}$ content of the formulated fertilizer.
Table 1. High and low values for the analysis of the MAA polymerization.

\begin{tabular}{ccc}
\hline FACTORS & HIGH & LOW VALUE \\
& VALUE & \\
\hline Polymerization Temp. & $50^{\circ} \mathrm{C}$ & $90^{\circ} \mathrm{C}$ \\
Polymerization Time & $30 \mathrm{mins}$ & $90 \mathrm{mins}$ \\
K Loading Conc'n & $1000 \mathrm{ppm}$ & $4000 \mathrm{ppm}$ \\
\hline
\end{tabular}

\subsection{Preparation of CS-PMAA nanoparticle}

For the preparation of the CS-PMAA, polymerization time and temperature were varied following the experimental design generated to identify if these two factors are significant.

The formulation of the nanoparticles was done using a two-step process. The first step is the dissolution of chitosan in methacrylic acid (MAA) containing $250 \mathrm{ppm}$ of Mono Methyl Ether of Hydroquinone (MEHQ) as inhibitor while the second step is the polymerization of methacrylic acid. This polymerized MAA will then be radically polymerized to the chitosan forming the CSPMAA nanoparticle.

In the first step, $0.2 \% \mathrm{w} / \mathrm{v} \mathrm{CS}$ with $85 \%$ deacetylation was dissolved in a $0.5 \% \mathrm{v} / \mathrm{v}$ MAA aqueous solution for $12 \mathrm{~h}$ under the shaker at $300 \mathrm{rpm}$ and $25^{\circ} \mathrm{C}$. For the second step, $0.2 \mathrm{mmol}$ of $\mathrm{K}_{2} \mathrm{~S}_{2} \mathrm{O}_{8}$ was added to the solution to start the polymerization of MAA. The polymerization time and temperature were varied with center points of 60 ${ }^{\circ} \mathrm{C}$ polymerization temperature and $60 \mathrm{mins}$ polymerization time. The high and low values used were shown in Table 1.

\subsection{Entrapment of $\mathrm{K}^{+}$in the CS-PMAA}

After preparation of nanoparticle carrier, varying amounts of MOP was dissolved in the nanoparticle solution. This solution was oven dried at $60^{\circ} \mathrm{C}$ for $8 \mathrm{hrs}$. The high and low values of the final $\mathrm{K}^{+}$concentration in the solution are 4000ppm $\mathrm{K}^{+}$and $1000 \mathrm{ppm} \mathrm{K}^{+}$, respectively, corresponding to the $2: 1$ and $1: 2$ ratio of $\mathrm{K}$ loading to the nanoparticle. These values are based on the $2000 \mathrm{ppm} \mathrm{K}^{+}$, 1:1 basis of the encapsulation of $\mathrm{K}$. These values were shown in Table 1.

\subsection{Characterization of K-CS-PMAA fertilizer formulated}

The characterization of the nanocarrier CS-PMAA and fertilizer formulated at optimum conditions was done in terms of total K content, size and morphology, chemical composition, and slow-release behavior.

The total $\mathrm{K}$ content of K-CS-PMAA fertilizer formulated was determined using Sherwood Flame photometer (Model 410) with sample preparation method of dry ashing. The size and morphology were analyzed using Zetasizer ZS90 or Dynamic Light Scattering (DLS) Particle Size and Zeta Potential Analyzer and Floor-top Scanning Electron Microscope (SEM). For the DLS, 
sample preparation of sonicating the sample for 15 minutes was done before the analysis with water as dispersant medium.

Shimadzu IR Prestige-21 Fourier Transform Spectrometer was used to analyze the chemical composition of CS-PMAA and K-CS-PMAA and to determine if there was an attachment of $\mathrm{K}^{+}$in the carrier.

\subsection{Slow release behavior analysis}

The slow release behavior of the fertilizer formulated was analyzed by simulating its release in a Britton-Robinson buffer solution.

The study was done by analyzing the release of $0.08 \mathrm{~g}$ of the K-CS-PMAA Fertilizer and MOP in a $1 \mathrm{~L}$ Robinson-Britton buffer. At certain time (0, 2, 4, 12, 24, and $48 \mathrm{hrs}$ ), $5 \mathrm{~mL}$ of the solution considered as leachate was obtained for analysis of the $\mathrm{K}$ content using Flame photometer. There are three set-ups for the release; (1) blank, (2) MOP as sample, and (3) K-CS-PMAA fertilizer formulated as sample.

\section{Results and discussion}

\subsection{Optimum conditions for K-CS-PMAA formulation}

The optimum condition of K-CS-PMAA fertilizer formulation was determined with polymerization time and $\mathrm{K}$ loading concentration as significant factors and polymerization temperature set at $50^{\circ} \mathrm{C}$. The polymerization temperature was determined as an insignificant factor for the response. The optimization analysis was run using Central Composite Design (CCD) with a center value of $2000 \mathrm{ppm} \mathrm{K}^{+}$loading concentration. The suggested model for the optimization study of K-CSPMAA formulation is a reduced quadratic model with forward regression elimination. The ANOVA table is shown in Table 2.

Table 2. Analysis of variance for optimization of conditions with final $\mathrm{K}$ content concentration as response using the RSMbased flask-level experiments.

\begin{tabular}{lccccc}
\hline \multicolumn{1}{c}{ SOURCE } & $\begin{array}{c}\text { SUM OF } \\
\text { SQUARES }\end{array}$ & DF & $\begin{array}{c}\text { MEAN } \\
\text { SQUARE }\end{array}$ & $\begin{array}{c}\text { F- } \\
\text { VALUE }\end{array}$ & P-VALUE \\
\hline Model & 833.91 & 4 & 208.48 & 34.50 & $<0.0001$ \\
$\begin{array}{l}\text { A- } \\
\text { Polymerization }\end{array}$ & 61.57 & 1 & 61.57 & 10.09 & 0.00063 \\
time & & & & & \\
B-K Loading & 587.67 & 1 & 587.67 & 96.27 & $<0.0001$ \\
$\begin{array}{l}\text { Concn. } \\
\mathbf{A}^{2}\end{array}$ & 31.35 & 1 & 31.35 & 5.14 & 0.0387 \\
$\mathbf{B}^{2}$ & 77.22 & 1 & 77.22 & 12.65 & 0.0029 \\
Residual & 91.57 & 15 & 6.10 & & \\
Lack of fit & 22.67 & 4 & 5.67 & 0.9049 & 0.4940 \\
$\begin{array}{l}\text { Pure error } \\
\text { Cor. Total }\end{array}$ & 68.90 & 11 & 6.26 & & \\
\hline
\end{tabular}

The model F-value is 34.5 , implying that the model is significant with $0.01 \%$ chance that the F-value this large can occur due to noise. The p-value with less than 0.0500 indicates the significant model terms. The Analysis of Variance showed that polymerization time and $\mathrm{K}$ concentration in the solution are significant with no interaction. The p-values of the two are 0.0104 and $<0.0001$, respectively.

The generated coded equation for the optimization of the K-CS-PMAA fertilizer formulation is shown in Equation 2. The relative impact of the significant factors can be identified and analyzed by comparing the sign and coefficient of the factors.

$$
\begin{aligned}
& K \text { Content Concentration } \\
& \qquad \begin{aligned}
& 25.43-1.96 A+6.06 B+1.85 A^{2} \\
& -2.91 B^{2}
\end{aligned}
\end{aligned}
$$

where $\mathrm{A}$ is the polymerization time in mins, $\mathrm{B}$ is the $\mathrm{K}$ loading concentration in ppm, $\mathrm{A}^{2}$ is the quadratic term of polymerization time, $\mathrm{B}^{2}$ is the quadratic term of $\mathrm{K}$ loading concentration. The equation shows that $\mathrm{K}$ loading concentration has the most significant effect between the two significant factors. The $\mathrm{K}$ loading has the direct effect to the $\mathrm{K}$ content concentration as represented by the positive sign values of 6.06 and 1.84 . On the other hand, the polymerization time has less effect compared to the $\mathrm{K}$ loading and inversely affect the $\mathrm{K}$ content concentration with in negative values of 1.96 and 2.91. The one-graph effect of significant factors was illustrated in Figure 1.

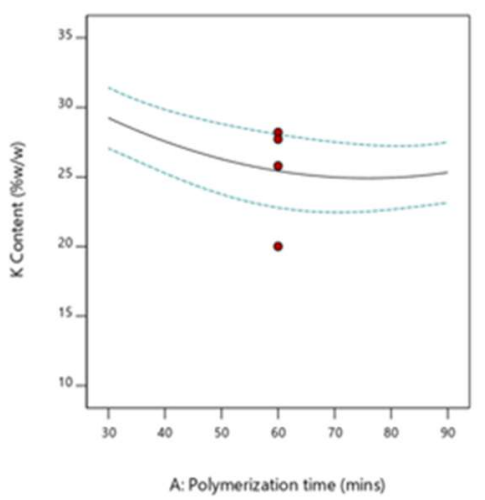

(a)

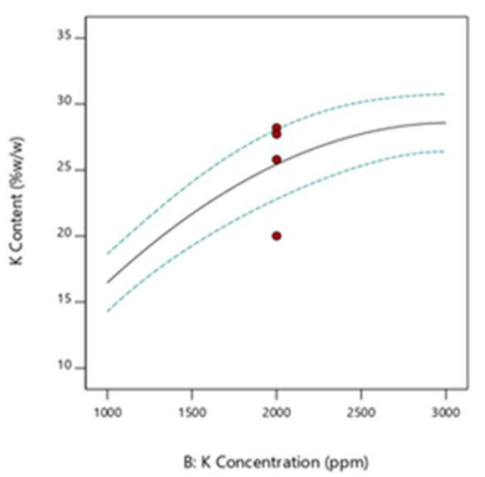

(b)

Fig.1. Response of the effect of (a) K concentration loaded and (b) polymerization time to the final $\mathrm{K}$ content concentration of the K-CS-PMAA fertilizer optimization study.

Numerical optimization was subsequently done to determine conditions for maximum $\mathrm{K}$ content of the formulated fertilizer. Table 3 shows the optimum conditions with 0.950 desirability. 
Table 3. Optimum conditions for K-CS-PMAA fertilizer formulation.

\begin{tabular}{lc}
\hline \multicolumn{1}{c}{ FACTOR } & OPTIMUM CONDITION \\
\hline K Loading Concentration, ppm & 3000 \\
Polymerization time, mins & 30 \\
\hline
\end{tabular}

The optimum conditions obtained were verified experimentally. The results of the experimental verification runs with three replicates for the $\mathrm{K}$ fertilizer formulation were summarized in Table 4.

Table 4. Summary of the experimental verification for K-CSPMAA fertilizer formulation.

\begin{tabular}{cc}
\hline TRIAL & $\begin{array}{c}\text { CONCENTRATION } \\
(\mathbf{\%} \text { w/w) }\end{array}$ \\
\hline 1 & 35.1143 \\
2 & 34.5088 \\
3 & 35.3100 \\
Actual Average & 34.9777 \\
Predicted Value & 32.3974 \\
Percent Difference, \% & 7.66 \\
\hline
\end{tabular}

The actual value of $34.98 \%$ obtained is within the $95 \%$ PI range of 28.60 to 36.20 with absolute percent difference of $7.66 \%$ of that of predicted value. It was also confirmed that at higher axial points the $\mathrm{K}$ content concentration showed a decrease in the $\mathrm{K}$ content concentration. Thus, indicating that the carrier cannot hold more than $3000 \mathrm{ppm}$ and validating that the model obtained for the optimization was valid.

\subsection{Effect of process conditions on total $\mathrm{K}$ content of the formulated K-CS-PMAA fertilizer}

\subsubsection{Effect of $K$ loading concentration}

Based from Equation 1, the $\mathrm{K}$ loading concentration is the most significant factor in increasing the content of K-CSPMAA fertilizer formulated, the more MOP added to the solution the higher the concentration of $\mathrm{K}$ in the solution. However, only a significant amount of $\mathrm{K}^{+}$can be entrapped in the nanocarrier. An observable precipitation occurs at excessive amount of $\mathrm{K}$ loading.

In this study, a K loading concentration of 2000ppm $\mathrm{K}^{+}$was used as the 1:1 ratio for the K:CS-PMAA that is based from the preliminary study on the entrapment of $\mathrm{K}$ fertilizer alone made by Eraña (2015). 2000 ppm K${ }^{+}$was used to incorporate in the nanocarrier and still no precipitation was observed only a noticeable change in color. This value corresponds to the equal amount of the mass $\mathrm{K}^{+}$and chitosan present in the solution.

In the study of Corradini and his colleagues (2010) on the incorporation of NPK fertilizer to CS-PMAA, the amount of $\mathrm{K}^{+}$incorporated was made up to the final concentration of $400 \mathrm{ppm} \mathrm{K} \mathrm{K}^{+}$. This is due to the observed precipitation of the colloidal suspension upon addition of greater than $400 \mathrm{ppm} \mathrm{K} \mathrm{K}^{+}$[2]. Similar study was conducted by Hasaneen and colleagues (2014) and same observation were inferred. In addition, Hasaneen discussed that at higher concentration the stability of the solution was affected.

The addition of higher concentration than 2000ppm $\mathrm{K}^{+}$did not result to observable precipitation, however the intensity of color pink increased. This color corresponds to the fillers present in the MOP beside the $\mathrm{K}$ alone.

\subsubsection{Effect of polymerization time}

The negative value of the polymerization time in Equation 1 proved that this factor inversely affects the $\mathrm{K}$ content concentration. The main factor that determines the polymerization time is the swelling behavior of CS. Carreira and collegueas (2010) explained that the swelling has both positive and negative effects. The swelling of chitosan-based polymers allow increase in the surface area therefore permitting greater amount of loading. However, as it increases its size it also allows fast release of the loaded particle. Furthermore, Szymańska and Winnicka (2015) discussed that the stability of CS decreases at prolong time. Thus, since the radical polymerization of chitosan and PMAA happens in the aqueous solution at prolong time, the CS swells simultaneously as the MAA polymerized causing inhibition of the attachment of more PMAA into CS [5].

\subsubsection{Effect of polymerization temperature}

The polymerization temperature was found to be insignificant at the bounds experimented upon. In the study of Zhang and Peppas (2000), they determine the effect of polymerization temperature of MAA as it attached to CS and found that the temperature affects the reaction at certain interval. The effect of temperature was shown in Figure 2 [6].

It was observed, at lower temperatures starting from $30^{\circ} \mathrm{C}$ at $\mathrm{pH} 4.05$ and $40^{\circ} \mathrm{C}$ at $\mathrm{pH} 6.16$, the swelling ratio (ratio of hydrated volume to dry volume) of PMAA is high compared to higher temperature. Zhang and Peppas (2000) discussed that for PMAA, the effect of polymerization temperature decreases at increasing temperature until it reaches the lower critical solution temperature (LCST). Beyond this value, the swelling ratio becomes constant.

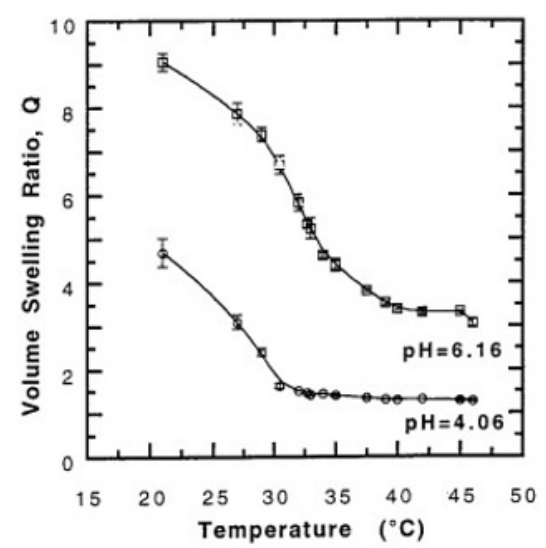

Fig.2. Effect of temperature to the swelling behavior of PMAA/PNIPAAm at $\mathrm{pH} 4.06$ and 6.16 
Source: Zhang and Peppas, 2000

This is the reason for the insignificance of the polymerization temperature in the experiment since the bounds set was at low value of $50^{\circ} \mathrm{C}$ which expects to have a constant result of its effect to swelling even there will be an increase.

\subsection{Characterization of K-CS-PMAA fertilizer formulated}

\subsubsection{Particle size and morphology distribution}

The size distribution intensity of the K-CS-PMAA fertilizer was analyzed using DLS as shown in Figure 3 while the zaverage potential was summarized in Table 5. The peaks of the sizes identified the composition of the nanoparticle sizes. The analysis was run for three times projecting 2 observable peaks with one peak that is more prominent than the other.

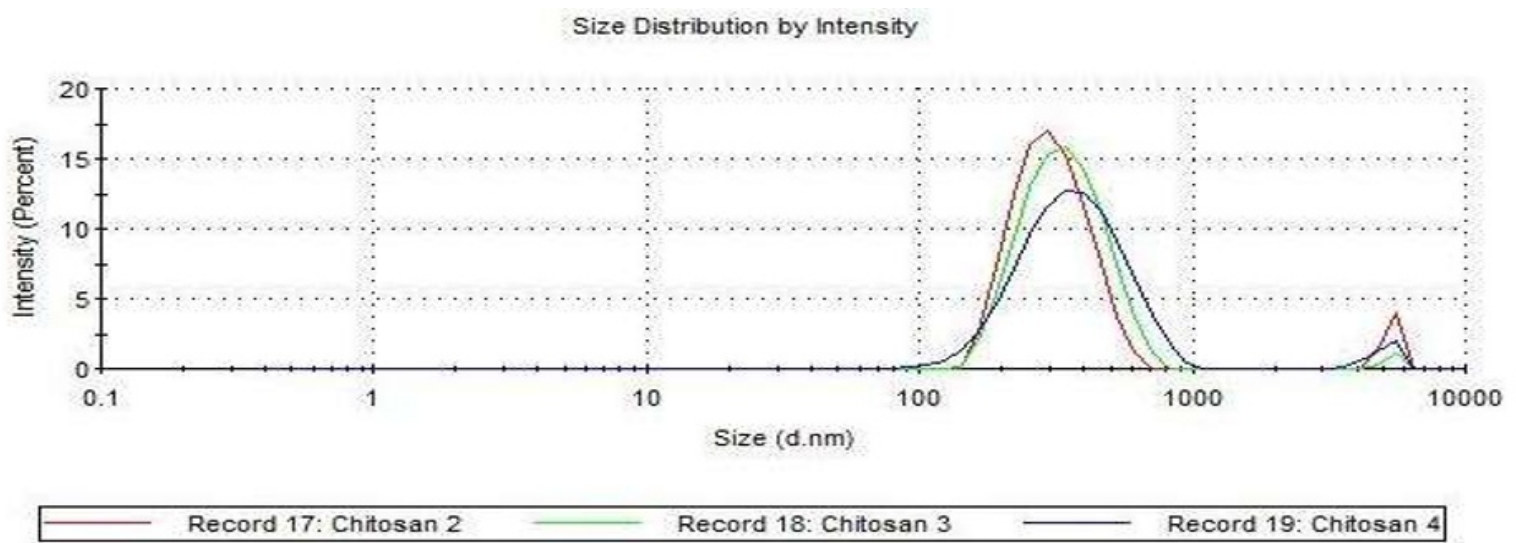

Fig.3. Size distribution by intensity of K-CS-PMAA Fertilizer formulated.

Table 5. Summary of the z-average of the K-CS-PMAA Fertilizer using DLS.

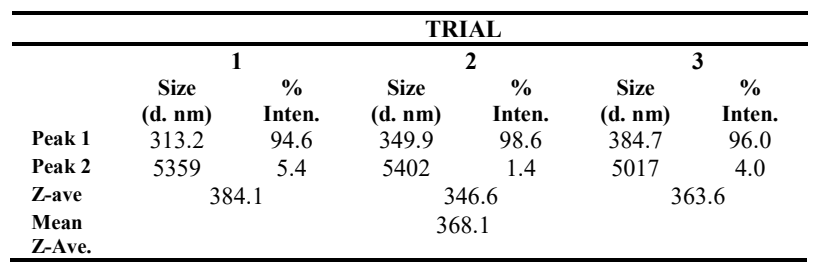

Table 6, on the other hand, summarizes the particle sizes detected by SEM while Figure 4 shows the morphology of the formulated fertilizer.

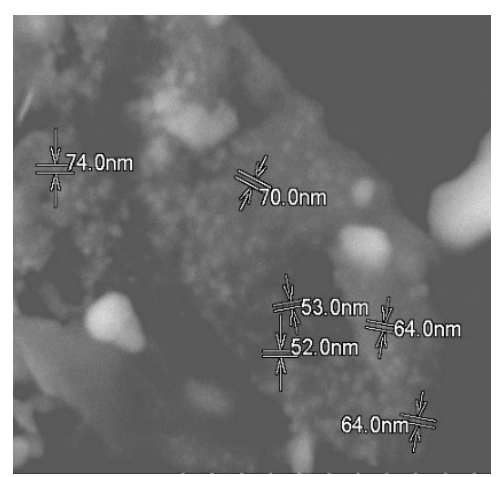

Fig.4. Sizes of the K-CS-PMAA Fertilizer approximated using SEM.

affecting the dispersion of the light as is hits the particle, reading the agglomerate as one individual particle.
Table 6. Approximate sizes of the nanoparticle using SEM.

\begin{tabular}{cc}
\hline Reading No. & Size (nm) \\
\hline 1 & 74.0 \\
2 & 70.0 \\
3 & 53.0 \\
4 & 52.0 \\
5 & 64.0 \\
6 & 64.0 \\
Average & 75.4 \\
\hline
\end{tabular}

For a particle to be considered in nanosized, it should have a diameter between 1-100 $\mathrm{nm}$, but can reach up to $1000 \mathrm{~nm}$ for nanopolymer. Based from the results in DLS, the fertilizer formulated has a mean z-average of 368.1 $\mathrm{nm}$. However, for the analysis using SEM the size ranges from 52.0-74.0 $\mathrm{nm}$ with an average of $75.4 \mathrm{~nm}$. Thus, based from the result, the fertilizer is in nanoscale.

The difference in particle sizes detected by DLS and SEM may be attributed to the sample preparation prior to analysis. Samples in the DLS were analyzed in suspension while samples in SEM was analyzed at its powdered form. As discussed, chitosan-based polymer exhibits swelling behavior once prepared in aqueous. Considering that the dispersant medium used was water during DLS, swelling might be the factor. Also, since DLS measures a particle using light scattering, agglomeration may had occurred during dispersion 
According to Corradini (2010), the mean diameter of the CS-PMAA nanoparticles in the dry state was approximately $78 \pm 1.5 \mathrm{~nm}$ and upon suspension the diameter of the nanoparticle increases to approximately $142 \mathrm{~nm}$ and further increases with the addition of different compounds. Thus, further conforming the effect of swelling to the fertilizer.

\subsubsection{Chemical composition determination using FT-IR}

The chemical composition analysis was determined by generating the FT-IR spectra of the blank CS-PMAA nanocarrier and K-CS-PMAA fertilizer. The FT-IR spectra overlay of the CS-PMAA and K-CS-PMAA was shown in Figure 5.

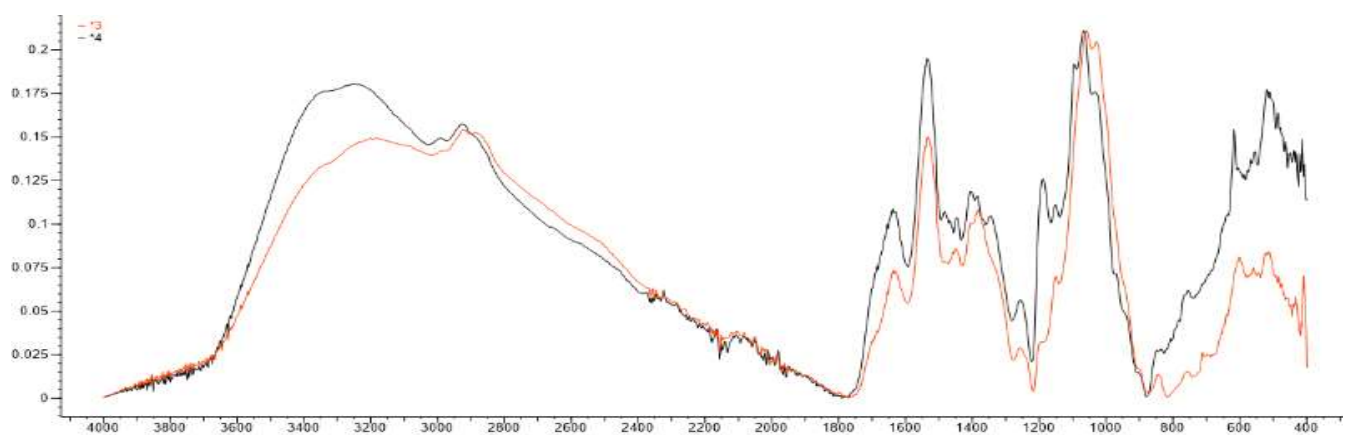

Fig.5. FT-IR spectra overlay of CS-PMAA (orange) and K-CS-PMAA fertilizer (black).

The FT-IR spectra of the blank and the K-CS-PMAA fertilizer showed the presence of CS-PMAA as confirmed by the functional groups read based on its wavenumber. The overlay of both spectrum showed a higher peak for the loaded with K-CS-PMAA fertilizer compared with the blank. The functional groups assignment for the characteristic peaks was shown in Table 7 and 8 .

Table 7. Functional group assignment for the characteristic peaks of CS-PMAA nanoparticle.

\begin{tabular}{|c|c|}
\hline $\begin{array}{c}\text { Wavenumber } \\
\left(\mathrm{cm}^{-1}\right)\end{array}$ & Functional Group Assignmen \\
\hline 3201.31 & Hydroxyl and N-H groups stretch \\
\hline 2923.60 & $\begin{array}{l}\mathrm{C}-\mathrm{H} \text { stretching of methylene and } \\
\text { methyl groups of glycol CS }\end{array}$ \\
\hline 1637.29 & $\begin{array}{l}\text { Carbonyl and amino group of the } \\
\text { aminoacetyl group of CS }\end{array}$ \\
\hline 1535.08 & Saccharide structure of CS \\
\hline 1382.73 & $\mathrm{C}-\mathrm{H}$ vibrations \\
\hline 1257.38 & Carbonyl group of PMAA \\
\hline 1028.82 & C-O stretch \\
\hline
\end{tabular}

Table 8. Functional group assignment for the characteristic peaks of CS-PMAA nanoparticle.

\begin{tabular}{cl}
\hline $\begin{array}{c}\text { Wavenumber } \\
\left(\mathbf{c m}^{-\mathbf{1}}\right)\end{array}$ & \multicolumn{1}{c}{ Functional Group Assignment } \\
\hline 3245.66 & $\begin{array}{l}\text { Hydroxyl and N-H groups stretch } \\
\text { C-H stretching of methylene and } \\
\text { methyl groups of glycol CS } \\
\text { Carbonyl and amino group of the } \\
\text { aminoacetyl group of CS } \\
\text { Saccharide structure of CS } \\
1637.29\end{array}$ \\
1535.08 & $\begin{array}{l}\text {-COO group of chitosan nanoparticles } \\
\text { and } \mathrm{K}^{+} \\
\text {-COO group of chitosan nanoparticles } \\
\text { and K }\end{array}$ \\
1483.01 & $\begin{array}{l}\text { Saccharide structure of CS } \\
\text { C-O stretch }\end{array}$ \\
1405.87 &
\end{tabular}

As observed, the two spectra are close to identical, however, an observable change at 1483.01 and 1405.87 $\mathrm{nm}$. These wavenumbers both corresponds to the $\mathrm{COO}^{-}$ stretch which became more emphasized upon addition of MOP because of its interaction with $\mathrm{K}^{+}$groups.

\subsection{Slow-release behaviour analysis}

The slow release behavior was done by analyzing the release of the fertilizer to $1 \mathrm{~L}$ of Robinson-Britton buffer. A buffer was used instead of water to account for the possible change in $\mathrm{pH}$ upon introduction of the fertilizer to the release medium.

The K-CS-PMAA fertilizer formulated was compared to nutrient release of the fertilizer grade, MOP. The concentration of the sample, MOP and K-CS-PMAA fertilizer was measured using flame photometer to determine the expected final concentration of the complete release. The expected concentration upon complete release has a value of 35.41 and $28.21 \mathrm{ppm}$ for MOP and K-CS-PMAA, respectively. Sampling was done for $0,2,4,12,24$, and $48 \mathrm{hrs}$ and the results was showed in Figure 6.

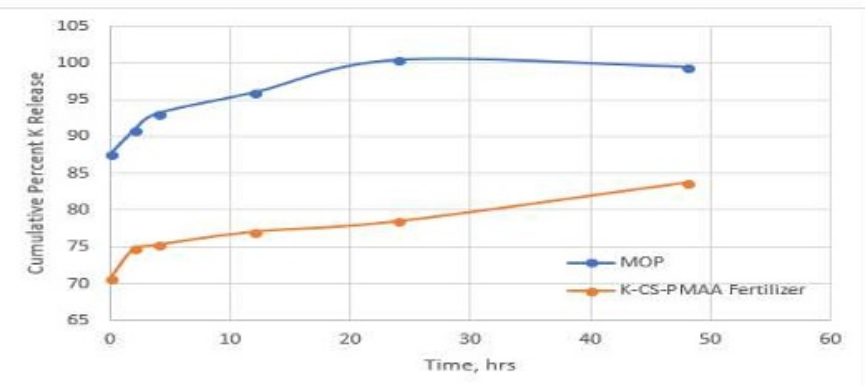

Fig.6. Cumulative percent release of $\mathrm{K}$ for slow release behavior determination.

As observed, the amount of $\mathrm{K}$ released in MOP was higher than the formulated K-CS-PMAA fertilizer. The 
$87.545 \%$ release value of MOP upon addition to the buffer was not yet achieved by the K-CS-PMAA fertilizer even after 48 hours. Therefore, the K-CS-PMAA is of slow release compared to the fertilizer grade, MOP. Please include criteria for slow release fertilizer

\section{Conclusion}

This study, the entrapment of potassium fertilizer into CSPMAA carrier posed a positive impact on its used for agricultural purposes. Nanofertilizer has the potential of reducing the environmental concerns of extensive use of fertilizer because of its slow release mechanism. In which because of its nanosized, it can be applied to the leaf surfaces entering the stomata during the gas uptake of the plant and thus avoiding direct interaction with soil systems [1].

In this study, parametric, optimization and characterization of the optimized conditions were done to for the formulation of potassium fertilizer entrapped in the CS-PMAA nanocarrier with final $\mathrm{K}$ content concentration as the response. The significant factors out of three factors varied is the polymerization time and $\mathrm{K}$ loading concentration with optimized conditions of $3000 \mathrm{ppm} \mathrm{K} \mathrm{K}^{+}$ polymerized for 30 mins. Relative high amount of $34.98 \%$ $\mathrm{K}$ content concentration was formulated using the optimized process conditions entrapment and proved to be in nanosized. Furthermore, the slow release behavior comparison with the fertilizer grade, MOP indicated that the fertilizer formulated is better for exhibiting slow release mechanism for the uptake of the plants.

This study focused on the one response only, final $\mathrm{K}$ content concentration. for the optimization, however, other factors can be taken into consideration that can affect the slow-release behavior of the fertilizer formulated and its size. Other factors to consider are the swelling effect and medium of release of the fertilizer upon application in the solution. It was found that application of the chitosan-based polymers in aqueous solution increases its size thus competing with the goal of achieving nanosized and allows immediate release of the nutrient. Also, the medium of release is an essential factor in determining the kinetics of it release. The medium used is a buffer solution and it was found that even though it allows change in $\mathrm{pH}$ upon application to disregard the effect of $\mathrm{pH}$ in the release behavior, the buffer can also be a factor to increase the dissociation rate of the $\mathrm{K}$ nutrient. Thus, it is highly recommended to determine the kinetic of release of the fertilizer formulated in different types of soil and account for the change in $\mathrm{pH}$.

\section{References}

\section{Journal Papers}

[1] H.M.M. ABDEL-AZIZ, M.N.A HASANEEN, \& A.M. OMER (2016). Nano chitosan-NPK fertilizer enhances the growth and productivity of wheat plants grown in sandy soil. Spanish Journal of Agricultural Research, 14. doi: 10.5424/sjar/2016141-8205.
[2] E. CORRADINI, M.R. DE MOURA \& L.H.C. MATTOSO (2010). A preliminary study of the incorporation of NPK fertilizer into chitosan nanoparticles. eXPRESS Polymer Letters, 4(8), 509-515. doi: 10.3144/expresspolymlett.2010.64

[3] C.L. DE VASCONCELOS et al. (2006). Effect of molecular weight and ionic strength on the formation of polyelectrolyte complexes based on poly(methacrylic acid) and chitosan. Biomacromolecules 7, 1245-1252.

[4] P. SOLANKI et al. (2015). Nano-fertilizers and their smart delivery system. In M. Rai et al. (Ed), Nanotechnologies in Food and Agriculture. doi: 10.1007/978-3-319- 14024-7 4

[5] E. SZYMAŃSKA \& K. WINNICKA (2015). Stability of chitosan - A challenge for pharmaceutical and biomedical applications. Mar Drugs, 13(4), 1819-1846. doi: 10.3390/md13041819.

[6] J. ZHANG \& N.A. PEPPAS (2000). Synthesis and characterization of $\mathrm{pH}^{-}$and temperaturesensitive $\operatorname{poly}(m e t h a c r y l i c \quad a c i d) / \operatorname{poly}(N-$ isopropylacrylamide) interpenetrating polymeric networks. Macromolecules, 2000, 33 (1), pp 102107. doi: $10.1021 / \mathrm{ma991398q}$.

Unpublished Dissertations

[7] C.M.D. ERAÑA (2015). Formulation and characterization of nano-potassium fertilizer and its effect on the growth of corn (Zea mays L.) IPB Var. 6. Unpublished undergraduate thesis, CAFS, UPLB. 